\title{
BRIDGING RADIAL AND NON-RADIAL MEASURES OF EFFICIENCY IN DEA
}

\author{
Necmi Kemal Avkiran \\ UQ Business School, The University of Queensland \\ 11 Salisbury Road, Ipswich, QLD4305 Australia \\ n.avkiran@business.uq.edu.au \\ Kaoru Tone* \\ National Graduate Institute for Policy Studies \\ 7-22-1 Roppongi, Minato-ku, Tokyo 106-8677, Japan \\ tone@grips.ac.jp \\ Miki Tsutsui \\ Central Research Institute of Electric Power Industry \\ 2-11-1 Iwado Kita, Komae-shi, Tokyo 201-8511, Japan \\ miki@criepi.denken.or.jp
}

* Corresponding author. 


\title{
BRIDGING RADIAL AND NON-RADIAL MEASURES OF EFFICIENCY IN DEA
}

\begin{abstract}
Data envelopment analysis (DEA) has been utilized worldwide for measuring efficiencies of banks, telecommunications, electric utilities and so forth. Yet, the existing models have some well known shortcomings that limit their usefulness. In DEA we have two fundamental approaches to measuring efficiency with very different characteristics; radial and non-radial. We demonstrate a method for linking these two approaches in a unified framework called Connected-SBM. It includes two scalar parameters, and by changing the parameter values we can relocate the analysis anywhere between the radial and the non-radial models. An appropriate choice of these parameters can overcome the key shortcomings inherent in the two approaches, namely, proportionality and mixed patterns of slacks.
\end{abstract}

Keywords: proportionality; slacks; DEA; CCR; SBM 


\section{Introduction}

Data envelopment analysis (DEA) has been under development since the seminal paper by Charnes, Cooper, and Rhodes (1978). The many theoretical developments of DEA over the years have been accompanied by an equally impressive application of the technique across a wide range of disciplines (see Gattoufi, Oral and Reisman, 2004, for a thorough literature review). Given the widespread use of DEA in relative efficiency / productivity measurement, an area close to the hearts and minds of managers as well as policy makers, it is important to pause and scrutinise the state of the art.

In DEA, we have two measures of efficiency with different characteristics; radial and non-radial. Historically, the radial models, represented by the CCR model (Charnes et al., 1978), was the first DEA model, whereas the non-radial models, represented by the SBM model (slacks-based measure by Tone, 2001) was a late comer. ${ }^{1}$ In the input-oriented case, the CCR deals mainly with proportionate reduction of input resources. For example, if a DMU (decision making unit) has two inputs, this model aims at obtaining the maximum rate of reduction with the same proportion, i.e. a radial contraction in the two inputs that can produce the current outputs.

In contrast, the non-radial models put aside the assumption of proportionate contraction in inputs and aim at obtaining the maximum rate of reduction in inputs that may discard varying proportions of the original input resources. In this paper we demonstrate that the two approaches can be bridged by manipulating two parameters, and that by choosing these parameters appropriately, we can generate what we call, the 'Connected' models.

Ultimately, our objective is to present a new approach based on a synthesis of existing theory that does not improvise efficiency measurement. Motivated by this objective, we demonstrate how the proportionality of slacks can be controlled to suit the needs of the user, and how the problem of mixed slacks values can be addressed to open the way for a healthier co-existence of DEA with parametric techniques.

The remainder of this paper unfolds as follows. In the next section, we introduce the CCR and the SBM models, and point out their shortcomings. In section 3, we propose a Connected-SBM model that links the two approaches. We demonstrate a numerical example in section 4. Then we extend the Connected-SBM

\footnotetext{
${ }^{1}$ Additive DEA models can also directly measure non-radial inefficiency but are unable to report the efficiency of the unit in a scalar value. As such, SBM is regarded as the successor of additive models.
} 
model to other orientations, to variable returns-to-scale, and to weighted situations in section 5 . We summarize the results and conclude the paper in the last section.

\section{Representative Radial and Non-Radial Measures of Efficiency and Their Shortcomings}

In this section we introduce the CCR and SBM models as representative radial and non-radial measures of efficiency respectively, and point out their shortcomings. Throughout this paper, we deal with $n$ DMUs $(j=1, \ldots, n)$ having $m$ inputs $(i=1, \ldots, m)$ and $s$ outputs $(r=1, \ldots, s)$. The input and output matrices are denoted by $X=\left\{x_{i j}\right\} \in R^{m \times n}$ and $Y=\left\{y_{r j}\right\} \in R^{s \times n}$, respectively. We assume $X>0$ and $Y>0$.

\subsection{The CCR Model}

The input-oriented CCR model evaluates the efficiency $\theta^{*}$ of DMU $\left(x_{o}, y_{o}\right)$ by solving the following linear program:

[CCR-I]

$\theta^{*}=\min _{\theta, \mu, t^{-}} \theta$

subject to

$$
\begin{aligned}
& \theta x_{o}=X \mu+t^{-} \\
& y_{o} \leq Y \mu \\
& \mu \geq 0, t^{-} \geq 0 .
\end{aligned}
$$

where $\mu$ represents the intensity vector and $t^{-}$denotes the non-radial slacks.

Usually, we solve [CCR-I] in a two phase process. In the first phase, we solve [CCR-I] and obtain $\theta^{*}$ (weak efficiency). At this stage, we define the radial input slacks $s^{-^{*}}$ as $s^{-*}=\left(1-\theta^{*}\right) x_{o}$.

Then, in the second phase, we maximize $\sum_{i=1}^{m} t_{i}^{-} / x_{i o}$ in terms of $\mu$ and $t^{-}$, subject to $\theta^{*} x_{o}=X \mu+t^{-}$, (3) and (4). Let an optimal solution of this phase be $\left(t^{-*}, \mu^{*}\right)$. Then $t^{-*}$ denotes the non-radial slacks remaining after deletion of the radial slacks $s^{-*}$. In this way, we have the total slacks $\bar{s}^{-*}$ of the [CCR-I] 
model defined as the sum of $s^{-*}$ and $t^{-*}$ i.e. $\bar{s}^{--^{*}}=s^{-^{*}}+t^{-*}$. While any non-radial projections are reported along with radial projections, non-radial slacks are not reported in the scalar $\theta^{*}$.

\subsection{The SBM Model}

The input-oriented SBM model under the constant returns-to-scale assumption evaluates the efficiency $\rho^{*}$ of DMU $\left(x_{o}, y_{o}\right)$ by solving the following linear program:

[SBM-I-C]

$$
\rho^{*}=\min _{z^{-}, \lambda} 1-\frac{1}{m} \sum_{i=1}^{m} \frac{z_{i}^{-}}{x_{i o}}
$$

Subject to

$$
\begin{aligned}
& x_{o}=X \lambda+z^{-} \\
& y_{o} \leq Y \lambda \\
& \lambda \geq 0, z^{-} \geq 0
\end{aligned}
$$

where $\lambda$ is the intensity vector, and $z^{-}$represents non-radial input slacks.

\subsection{Comparison of Radial and Non-radial Inefficiencies}

We have already indicated that the models such as the CCR report only radial inefficiency in scalar $\theta^{*}$. Similarly, we have shown in the previous section that SBM models measure only non-radial inefficiency. For the SBM score $\rho^{*}$ to be less than 1 , there are two possible states for the radial efficiency score $\theta^{*}$. One possible combination of $\rho^{*}(<1)$ and $\theta^{*}$ is where there are radial and non-radial slacks, which means $\theta^{*}$ will be less than 1 as well. The other possibility is the situation where the DMU is radially efficient (i.e. $\left.\theta^{*}=1\right)$ but there are some non-zero non-radial slacks $\left(t^{-*}\right)$.

\subsection{Shortcomings of the CCR model}

The main shortcoming of the CCR model is the neglect of non-radial slacks $t^{{ }^{*}}$ in reporting of the efficiency score $\theta^{*}$. In many cases, we find a lot of remaining non-radial slacks. So, if these slacks have an important role in evaluating managerial efficiency, the radial approaches may mislead the decision when we 
utilize the efficiency score $\theta^{*}$ as the only index for evaluating performance of DMUs. For instance, in manufacturing, inputs are determined by stringent product design parameters and reduction of an input as part of a benchmarking drive by management is likely to lead to a reduction of at least one other input e.g. a car that is re-designed with a lighter chassis can be re-fitted with lighter tires and less anti-pollution equipment as a result of a smaller engine that can be used to power the lighter car. Of course, it is difficult to argue that the input reductions in the above example will be proportional. Thus, in such a case, use of a radial approach to efficiency analysis will be inappropriate.

\subsection{Shortcomings of the SBM model}

Since non-radial models such as SBM capture the slacks directly, the optimal efficiency value $\rho^{*}$ accounts for the non-radial slacks which are not considered in the radial models, and generally we have $\rho^{*} \leq \theta^{*}$ (see Tone, 2001).

The SBM-projection to the efficient frontier is defined by $\hat{x}_{o}=x_{o}-z^{-*}$. Thus, the projected DMU may lose the proportionality in the original $x_{o}$. This is characteristic of the non-radial models and, if the loss of the original proportionality is inappropriate for the analysis, then this becomes a shortcoming for non-radial models. However, it is difficult to envisage such a case in practice. Here, we offer a simple example regarding bank branches. If the number of branches were to be reduced as part of a managerial cost-cutting exercise, then we would expect a somewhat proportional reduction in overall staff numbers for the bank (inevitably some staff will be relocated to other branches), as well as a reduction in ancillary services such as cleaning services that are normally outsourced.

This shortcoming of non-radial models causes a problem when we evaluate efficiency change over time. The non-zero pattern of slacks at time period $t$ may significantly differ from that of time period $t+1$. Thus, we will be unable to tell which pattern is reasonable. Especially, when we consider these slacks as the sources of inefficiency and apply further analyses such as statistical regressions based on the thus obtained slacks, this occurrence of zero and non-zero pattern may distort the interpretation of results.

However, a potentially more significant shortcoming of SBM arises from the nature of the linear programming solution, where the optimal slacks tend to exhibit a sharp contrast in taking positive and zero 
values. The zeros correspond to the non-basic slacks in the optimal basis. For an SBM-inefficient DMU, if the number of DMUs in the optimal basis is $k$, the number of slacks in the basis is $m+s-k$. This demonstrates that at least $k$ out of $m+s$ slack variables must be zero. See Appendix A for more detailed comparisons of the zero and non-zero patterns between radial and non-radial models.

\section{Bridging Radial and Non-Radial Models}

In an effort to resolve the above mentioned shortcomings, we propose an SBM model restricted with two non-negative parameters $L \quad(0 \leq L \leq 1)$ and $U(\geq 1)$ as follows.

[SBM-I-C $(L, U)]$

$$
\tau^{*}=\min _{f, z^{-}, v^{-}, \lambda} 1-f
$$

subject to

$$
\begin{aligned}
& f=\frac{1}{m} \sum_{i=1}^{m} \frac{z_{i}^{-}}{x_{i o}} \\
& L f \leq \frac{z_{i}^{-}}{x_{i o}} \leq U f(i=1, \ldots, m) \\
& x_{o}=X \lambda+z^{-}+v^{-} \\
& y_{o} \leq Y \lambda \\
& \lambda \geq 0, z^{-} \geq 0, v^{-} \geq 0 .
\end{aligned}
$$

where $v^{-}$denotes non-radial input slacks induced in accordance with the constraint (11).

In this model, $f$ is the average of the normalized slacks $\left\{z_{i}^{-} / x_{i o}\right\}$ and the constraint (11) restricts each normalized slack $z_{i}^{-} / x_{i o}$ to the range $[L f, U f]$. Alternatively, $f$ can be described as the mean non-radial input inefficiency for the observed DMU. Thus, depending on the lower bound $L$ and upper bound $U$, the deviations from the average value $f$ are limited accordingly.

We have:

\section{[Lemma 1]}

The optimal objective value $\tau^{*}$ is increasing in $L$ and decreasing in $U$. Furthermore, $\tau^{*}$ is units-invariant. 
Below we demonstrate that this restricted SBM model includes the radial [CCR-I] and the non-radial [SBM-I-C] as special cases.

\section{[Theorem 1]}

If $L=1$ or $U=1$, then [restricted SBM-I-C $(1,1)]$ reduces to [CCR-I], i.e. $\tau^{*}=\theta^{*}$.

Proof: Suppose that $L=1$. Then we have

$$
\frac{z_{i}^{-}}{x_{i o}} \geq f=\frac{1}{m} \sum_{k=1}^{m} \frac{z_{k}^{-}}{x_{k o}}(\forall i)
$$

This leads to

$$
\frac{z_{i}^{-}}{x_{i o}}=f \text { and hence } z_{i}^{-}=f x_{i o}(\forall i)
$$

Then [restricted SBM-I-C $(1,1)]$ becomes

$$
\tau^{*}=\min 1-f
$$

subject to

$x_{o}=X \lambda+f x_{o}+v^{-}$

$y_{o} \leq Y \lambda$

$\lambda \geq 0, v^{-} \geq 0$.

Replacing $1-f$ by $\theta$, we have [CCR-I].

Similarly, we can prove that the $U=1$ case coincides with [CCR-I].

\section{[Theorem 2]}

If $L=0$ and $U \geq m$, then [restricted SBM-I-C $(0, U)$ ] becomes [SBM-I-C].

Proof: When $L=0$, the left inequality of (11) is automatically satisfied. If $U \geq m$, the right inequality of (11) is also always satisfied since $f$ is the average of $\left\{z_{i}^{-} / x_{i o}\right\}$. Hence the constraint (11) vanishes. In this case, in the constraint (12), the term $v^{-}$vanishes since the objective function is decreasing in $z_{i}^{-}$. Thus, this case turns out to [SBM-I-C].

QED

The above two theorems demonstrate that the restricted SBM model includes [CCR-I] and [SBM-I-C] as special cases and that the valid ranges of $L$ and $U$ are $[0,1]$ and $[1, m]$, respectively. We name such restricted 
SBM that simultaneously addresses the shortcomings of CCR and SBM models, 'Connected-SBM'.

Let an optimal solution for [Connected-SBM-I-C $(L, U)$ ] be $\left(\tau^{*}, f^{*}, \lambda^{*}, z^{-*}, v^{-*}\right)$. If we define the “Connected-SBM projection”2 by

$\hat{x}_{i o}=x_{i o}-z_{i}^{-*}(i=1, \ldots, m)$,

the normalized deviation of $\hat{x}_{i o}$ from the data $x_{i o}$ satisfies the inequalities

$L f^{*} \leq \frac{x_{i o}-\hat{x}_{i o}}{x_{i o}} \leq U f^{*}(\forall i)$

Thus, the relative deviation of the projected input from the original value is bounded by $L f^{*}$ and $U f^{*}$ for all inputs. Therefore, we can prevent the occurrence of positive and zero patterns or extremely unbalanced patterns often observed in the SBM models.

We can reduce these two parameters to a single parameter by setting

$U=m-(m-1) L$

In this one parameter setting, $L=0$ and $L=1$ correspond to [SBM-I-C] and [CCR-I], respectively. In accordance with the magnitude of $L$, the normalized slacks $\left\{z_{i}^{-} / x_{i o}\right\}$ tend to be uniform. In other words, a small $L$ permits relatively large variations of normalized slacks, while a large $L$ restricts them to a limited range.

\section{Numerical Example}

Table 1 shows 3 DMUs with two inputs $x_{1}$ and $x_{2}$, and one output $y$, and Figure 1 displays them graphically.

\section{[insert Table 1 about here]}

\section{[insert Figure 1 about here]}

DMUs A and B are on the efficient frontier and they have the full score in any DEA model. So, we observe the DMU C to see how its efficiency value changes in accordance with the lower $L$ and the upper $U$ values. In this case we employ the formula (17) and define $U=2-L$. Table 2 reports the optimal solutions of the

2 Although the "true" projection onto the efficient input-frontier is achieved by $X \lambda^{*}=x_{o}-z^{-*}-v^{-*}$, we use here an approximate projection. 
Connected-SBM model for $L=0,0.2,0.588,0.8$ and $1 . L=0$ and $L=1$ correspond to the non-radial SBM and the radial CCR models, respectively.

At $L=1, \mathrm{C}$ is radially reduced to $\mathrm{P}_{1}$ with $x_{1}{ }^{*}=3, x_{2}{ }^{*}=18.8$ in Figure 1 . The normalized deviations $z_{1}^{-*} / x_{1}$ and $z_{2}^{-*} / x_{2}$ are the same value 0.25 ; thus, the two inputs of $\mathrm{C}$ is proportionally reduced by $25 \%$. However, it still has a remaining non-radial slack, $v_{2}^{-*}=8.8$, against the efficient DMU A. At $L=0.8$, it moves to $\mathrm{P}_{2}$ with $x_{1}{ }^{*}=3, x_{2}{ }^{*}=15.6$, and we can see that it loses its original proportionality as designated by $z_{1}^{-*} / x_{1}=0.25$ and $z_{2}^{-*} / x_{2}=0.38$. At $L=0.588, \mathrm{C}$ is projected to A and $v_{2}^{-*}$ vanishes. At $L=0.2$, C is projected to $\mathrm{P}_{3}$ on the piecewise linear segment connecting $\mathrm{A}$ and $\mathrm{B}$. Then, finally at $L=0$, it is projected to $\mathrm{B}$ which is the point of SBM solution. The efficiency score decreases steadily from 0.75 (CCR) to 0.52 (SBM).

\section{[insert Table 2 about here]}

\section{Extensions}

We can extend this model to various situations, e.g. output-oriented, non-oriented, variable returns-to-scale, and the weighted environment.

\subsection{Output-oriented Model}

The output-oriented version can be represented as follows:

[Connected-SBM-O-C $(L, U)]$

$$
\tau^{*}=\max _{f, z^{+}, v^{+}, \lambda} 1+g
$$

subject to

$$
\begin{aligned}
g & =\frac{1}{s} \sum_{r=1}^{s} \frac{z_{r}^{+}}{y_{r o}} \\
L g & \leq \frac{z_{r}^{+}}{y_{r o}} \leq U g(r=1, \ldots, s) \\
x_{o} & \geq X \lambda
\end{aligned}
$$




$$
\begin{aligned}
& y_{o}=Y \lambda-z^{+}-v^{+} \\
& \lambda \geq 0, z^{+} \geq 0, v^{+} \geq 0 .
\end{aligned}
$$

\subsection{Non-oriented Model}

The non-oriented version can be defined by replacing the objective function by

$\min (1-f) /(1+g)$

We employ the constraints (10)-(12) and (19), (20) and (22), where the values of $L$ and $U$ vary from input to output. This fractional program can be transformed into an equivalent linear program using Charnes-Cooper transformation (see Tone, 2001 for details).

\subsection{Variable returns-to-scale Model}

The variable returns-to-scale model can be obtained by adding the following convexity condition on $\lambda$. $\lambda_{1}+\cdots+\lambda_{n}=1$.

\subsection{Weighted Model}

A weighted, input-oriented model can be defined by taking the weighted average of the normalized slacks as follows:

$f=\frac{1}{m} \sum_{i=1}^{m} \frac{w_{i} z_{i}^{-}}{x_{i o}}$

where $w_{i}(\geq 0)$ is the weight to input $i$ and satisfies

$\sum_{i=1}^{m} w_{i}=m$.

An input item with a large weight will exert influence over the average and hence over the lower and upper bounds.

\subsection{Individual Settings of Lower/Upper Bounds}

Although we utilized common lower/upper bounds in the Connected-SBM models, they can be defined 
individually by input items, i.e. $L_{i}$ and $U_{i}(i=1, \ldots, m)$. If some input allows only small variations in proportion, we can set $L_{i}$ and $U_{i}$ close to unity, and vice versa.

\section{Concluding Remarks}

This paper demonstrates a scheme for integrating the radial and non-radial DEA models in order to control the proportionality of slacks and address the potential problem of mixed zero/non-zero slacks patterns over time. The Connected-SBM model utilizes two parameters for restricting the variations of the normalized slacks in a specified range. The well-known CCR and SBM models are the two extreme cases of this model.

Researchers and practitioners of DEA are now able to match estimated contraction of resources and expansion of outputs in a given production system to take more realistic efficiency measures. Returning to the example of car manufacturing in section 2.4, the different proportions of changes estimated by engineers can now be approximated in efficiency measurement by experimenting with the parameters of the Connected-SBM model. Such an approach can, in turn, lead to more comparable and reliable measurements, thus enhancing decision-making in benchmarking.

Similarly, sensitivity analysis by changing the parameters of the Connected-SBM model can help overcome the potential problem of mixed value slacks that could otherwise confound other analyses that depend on slacks. A case in example is the group of multi-stage analyses that combine DEA with parametric methods such as Tobit regression and Stochastic Frontier Analysis (see Avkiran and Rowlands, 2006, and Liu and Tone, 2006).

Future topics include the determination of the two parameters for various empirical applications. 


\section{Appendix A: Zero and Non-zero Patterns in Input Slacks}

We compare the zero and non-zero patterns for the input slacks obtained in the optimal solutions of the two approaches.

\section{A.1 Radial Model Case}

In this case, the difference between the original input $x_{o}$ and the proportionally reduced input $\theta^{*} x_{o}$ defines its input (radial) slacks as, $s^{-*}=\left(1-\theta^{*}\right) x_{o}$. Thus, for a technically inefficient DMU, i.e. $\theta^{*}<1$, $s^{-*}$ is strictly positive.

Furthermore, if we delete any remaining non-radial slacks $t^{{ }^{*}}$ from the input $x_{o}$, we have the total slacks, i.e. the sum of radial and non-radial slacks $\bar{s}^{--^{*}}=s^{-*}+t^{-*}$ which is not less than $s^{-*}$. In any case, for a technically inefficient DMU, all elements of the optimal radial input slacks are positive, e.g. $[+,+,+, \ldots,+]$.

\section{A.2 Non-radial Model Case}

Contrary to the radial case, the input slacks for a non-radially inefficient DMU, i.e. $\rho^{*}<1$, have a mixed zero and non-zero pattern such as $[0,+, 0,+, \ldots, 0],[0,+,+, 0, \ldots,+]$ or some other combination. This is caused by the nature of the optimal solution of linear programs as explained below.

Generally, the optimal solution of a linear program corresponds to a vertex of the polyhedron spanned by its set of linear constraints. This vertex corresponds to the basic solution which has, in the SBM case, at most $(m+s)$ non-zero elements out of the total $(n+m+s)$ variables. Thus, we have $n=(n+m+s)-(m+s)$ non-basic variables which have the value zero at the optimum. Suppose that the number of DMUs in the optimal basis is $k(\geq 1)$. Then the number of slacks in the optimal basis is $(m+s-k)$. This demonstrates that at least $k$ out of $(m+s)$ slacks variables $\left(s^{-}\right.$and $\left.s^{+}\right)$must be zero. This is the reason why we inevitably have the zero and non-zero patterns in SBM slacks. 


\section{References}

Avkiran, N.K., and Rowlands, T. 2006. How to better identify the true managerial performance: state of the art using DEA. OMEGA The International Journal of Management Science, forthcoming.

Charnes, A., Cooper, W.W., and Rhodes, E., 1978. Measuring the efficiency of decision making units. European Journal of Operational Research 2(6), 429-444.

Gattoufi, S., Oral, M., and Reisman, A., 2004. Data envelopment analysis literature: a bibliography update (1951-2001). Socio-Economic Planning Sciences 38, 159-229.

Liu, J. and Tone, K., 2006. A multistage method to measure efficiency and its application to Japanese banking industry. Socio-Economic Planning Sciences, forthcoming.

Tone, K., 2001. A slacks-based measure of efficiency in data envelopment analysis. European Journal of Operational Research 130, 498-509. 


\section{Table 1}

Numerical Example

\begin{tabular}{lccc}
\hline DMU & $\mathrm{x}_{1}$ & $\mathrm{x}_{2}$ & $\mathrm{y}$ \\
\hline $\mathrm{A}$ & 3 & 10 & 1 \\
B & 4 & 1 & 1 \\
$\mathrm{C}$ & 4 & 25 & 1 \\
\hline
\end{tabular}




\section{Table 2}

Results of Connected-SBM Model

\begin{tabular}{|c|c|c|c|c|c|}
\hline$L$ & 0 & 0.2 & 0.588 & 0.8 & 1 \\
\hline$\tau^{*}$ & 0.52 & 0.54 & 0.58 & 0.69 & 0.75 \\
\hline$f^{*}$ & 0.48 & 0.46 & 0.42 & 0.31 & 0.25 \\
\hline Reference & B & A, B & A & A & $\mathrm{A}$ \\
\hline$z_{1}^{-*}$ & 0 & 0.37 & 1 & 1 & 1 \\
\hline$z_{2}^{-*}$ & 24 & 20 & 15 & 9.4 & 6.3 \\
\hline$z_{I}^{*} * / x_{1}$ & 0 & 0.1 & 0.25 & 0.25 & 0.25 \\
\hline$z_{2}^{*} * x_{2}$ & 0.96 & 0.83 & 0.6 & 0.38 & 0.25 \\
\hline$v_{l}^{-*}$ & 0 & 0 & 0 & 0 & 0 \\
\hline$v_{2}{ }^{*}$ & 0 & 0 & 0 & 5.6 & 8.8 \\
\hline Projection & B & P3 & A & P2 & P1 \\
\hline$x_{1}^{*}$ & 4 & 3.6 & 3 & 3 & 3 \\
\hline$x_{2} *$ & 1 & 4.3 & 10 & 15.6 & 18.8 \\
\hline
\end{tabular}




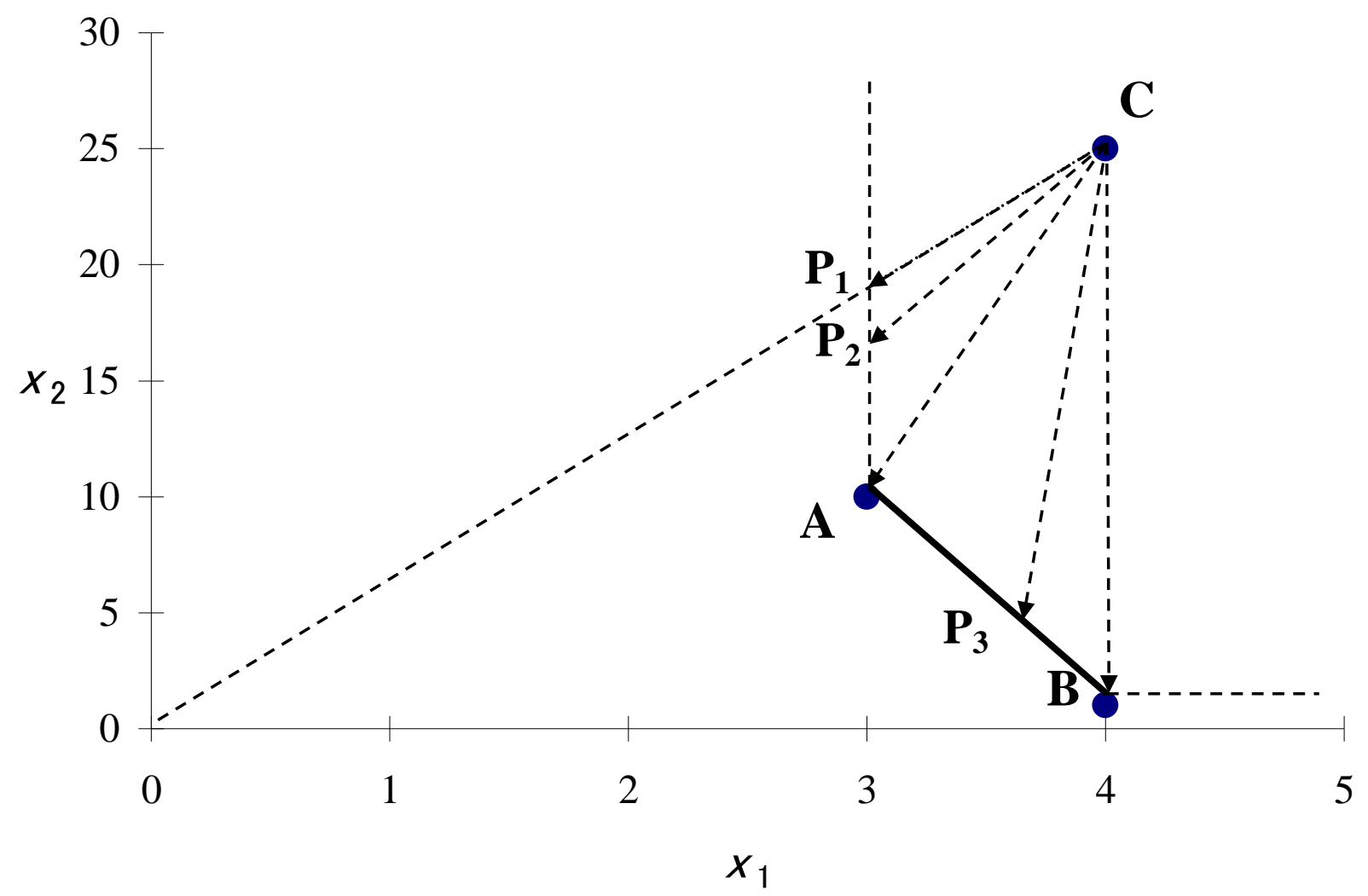

Fig. 1. Plot of 3 DMUs and Projections of C 